\title{
A Safe and Rapid Technique for Pacemaker Implantation: Roadmap-Guided Subclavian Vein Puncture
}

Hakan Gunes, Mahmut Tuna Katırcıbası, Akif Serhat Balcıoğlu, Ekrem Aksu, Abdullah Sokmen, Gulizar Sokmen, Murat Kerkutluoglu, Ahmet Çağrı Aykan, Sami Ozgul Department of Cardiology, Sutcu Imam University, Kahramanmaras, Turkey

ORCID:

Hakan Gunes: https://orcid.org/0000-0003-3853-5046

Mahmut Tuna KatırcıbasI: https://orcid.org/0000-0002-9871-492X

Akif Serhat Balcıoğlu: https://orcid.org/0000-0002-7979-838X

Ekrem Aksu: https://orcid.org/0000-0003-1939-1008

Abdullah Sokmen: https://orcid.org/0000-0001-7801-0063

Gulizar Sokmen: https://orcid.org/0000-0003-4440-1749

Murat Kerkutluoglu: https://orcid.org/0000-0003-1007-0574

Ahmet Çağıı Aykan: https://orcid.org/0000-0001-5793-7978

Sami Ozgul: https://orcid.org/0000-0002-2984-9249

\section{Abstract}

Objective: Widely used method is blinded puncture of subclavian vein, but the complication rate is high in this method. In this study, we aimed to demonstrate the effect of roadmap use during implantation of permanent pacemaker on the success rate, speed of puncture and complications. Methods: The study was designed as a prospective randomized controlled study. Totally, 125 devices were implanted to the patients included in the study, and 518 punctures were performed for implantation of these devices. 186 punctures were performed in roadmap group and 332 punctures were performed in conventional group. Two groups were compared with regard to clinical and demographic features, speed and success of puncture and complications. Results: Baseline characteristics were similar between groups. Median duration of intervention for each puncture was $27(15 / 46) \mathrm{s}$ in roadmap group and $56(30 / 100) \mathrm{s}$ in conventional group. The number of attempts for a successful puncture was detected as $1(1 / 2)$ in roadmap group and $2(2 / 4)$ in conventional group. Arterial puncture incidence was $10.3 \%$ in roadmap group and $37 \%$ in conventional group $(P<0.001$ for all). Considering complications, the incidence of pneumothorax and intramuscular puncture was seen lower significantly $(P=0.046$ and $P=0.006$, respectively). Conclusion: Number of attempts for successful puncture, time needed for successful puncture, number of arterial puncture and complication rate was significantly lower in patients undergoing pacemaker implantation by roadmap technique. Based on these data, roadmap technique may take the place of conventional method of puncture.

Keywords: Defibrillator, pacemaker, roadmap, subclavian puncture

\section{INTRODUCTION}

Subclavian venous access is preferred frequently for implantation of permanent pacemakers since it allows the implantation of multiple leads in a reasonable period. ${ }^{[1,2]}$ Procedural success and complication rate is closely related with the operator experience and the anatomy of the operation area. Blinded punctures performed with conventional method can cause various complications. In the case of failed punctures, repeated attempts increase the risk of complications such as pneumothorax,

Submission: 01-Feb-19 Revision: 20-Mar-19 Accepted: 15-Apr-19

\begin{tabular}{|l|l|}
\hline \multicolumn{3}{|c|}{ Access this article online } \\
\hline Quick Response Code: & Website: \\
& \\
http://www.ijcva.com
\end{tabular}

hemothorax, lung laceration, arteriovenous fistula, and injury to brachial plexus. ${ }^{[3,4]}$ Contrast venography-guided puncture of the subclavian vein has been used to increase the success rate of the procedure and to avoid the complications. In this method, venous anatomy has been visualized by $15-20 \mathrm{cc}$ of contrast agent

Address for correspondence: Asst. Prof. Hakan Gunes, Department of Cardiology, Kahramanmaras Sutcu Imam University School of Medicine, Kahramanmaras, Turkey. E-mail: drhakangunes83@hotmail.com

This is an open access journal, and articles are distributed under the terms of the Creative Commons Attribution-NonCommercial-ShareAlike 4.0 License, which allows others to remix, tweak, and build upon the work non-commercially, as long as appropriate credit is given and the new creations are licensed under the identical terms.

For reprints contact: reprints@medknow.com

How to cite this article: Gunes $\mathrm{H}$, Katırcıbası MT, Balcığlu AS, Aksu E, Sokmen A, Sokmen G, et al. A safe and rapid technique for pacemaker İmplantation: Roadmap-guided subclavian vein puncture. Int J Cardiovasc Acad 2019;5:86-91. 
injection made from the ipsilateral peripheral venous access and subclavian puncture has been performed under fluoroscopy. ${ }^{[5,6]}$ Punctures performed between costoclavicular ligament and subclavian muscle can cause lead fractures later on. ${ }^{[7]}$ It is difficult to avoid this complication in punctures achieved by conventional method in which extrathoracic and intrathoracic subclavian vein discrimination cannot be done. Similarly, the complication rate including lead fractures decreased in studies determining extrathoracic subclavian vein anatomy to avoid lead fractures ${ }^{[8,9]}$ In addition, ultrasonography-guided intervention of axillary vein also reduced lead fractures and other complications related with the subclavian venous puncture. ${ }^{[10]}$

Roadmap is an imaging technique that is created by converting the first image taken during injection to digital information and holding it in the device memory. It is important to visualize the vascular bed simultaneously that allows the positioning of the guide wires and catheters without need for repetitive contrast injections. The usage of roadmap increases the success rate and speed of the cannulation. ${ }^{[11]}$

In this study, we aimed to investigate the effect of roadmap use during the implantation of permanent pacemaker on the success rate and speed of venous puncture, and number of attempts for successful puncture as well as to various complications such as arterial puncture, intramuscular puncture, pneumothorax, and pocket hematoma.

\section{Methods}

\section{Patient selection}

The study was designed as a prospective randomized controlled study. Patients over 18 years old who were taken to catheterization laboratory for permanent pacemaker implantation through the subclavian vein were included in the study. Patients were divided into two groups: those who underwent roadmap-guided subclavian venous puncture and those who performed conventional subclavian venous puncture. Both puncture techniques performed separate puncture for each lead implantation. In both groups, demographic characteristics, the total number of punctures performed, number and type of inserted devices, time for successful punctures, time for single successful intervention, number of arterial punctures, clinical and laboratory data before and after the procedure, and complications after the procedure were recorded. Diabetes mellitus was defined as fasting blood glucose $\geq 126 \mathrm{mg} / \mathrm{dl}$ or taking antidiabetic medication. Hypertension was defined as systolic arterial blood pressure $\geq 140 \mathrm{mmHg}$ or diastolic blood pressure $\geq 90 \mathrm{mmHg}$ measured on three separate office visit or taking antihypertensive treatment. The pacemaker implantation decision was made by our heart team including electrophysiologists and heart failure experts in accordance with related guidelines of the European Society of Cardiology and pacemaker implantation was performed by the same electrophysiologist in both groups.

\section{Pacemaker implantation procedure}

All patients were questioned whether receiving antiaggregant and anticoagulant therapy before the procedure and if necessary, they were managed properly, and the treatments were recorded. The implantation area was cleared off skin hairs $24 \mathrm{~h}$ before the procedure. Nasal oxygen was given to the patients when needed. All patients were sedated by intravenous midazolam (1-2.5 $\mathrm{mg}$ ) to perform a smooth implantation. Flumazenil was kept for use when required. Appropriate antibiotic prophylaxis was given to the patients before the procedure. Two venous vascular accesses were established in all patients. One of them was placed on ipsilateral antecubital region with the proper venous catheter for delivering contrast material to generate a roadmap in the roadmap group.

Following skin preparation and local anesthesia, the skin incision was made $2 \mathrm{~cm}$ below and parallel to the clavicle, as the medial edge of incision ending in $1 / 3$ middle part of the clavicle. The length of incision was $4-6 \mathrm{~cm}$ for implantable cardioverter defibrillators (ICDs) and 3-4 cm for pacemakers. After progressing toward pectoralis muscle fascia by blunt and sharp dissection, a pocket proper for device size was created under the muscle to decrease the erosion and to get a favorable appearance cosmetically based on the operator's choice. Then, in conventional subclavian venous puncture group, $2-3 \mathrm{ml}$ saline was taken by conventional 18 -gauge needle mounted to $10 \mathrm{cc}$ injector and the puncture was performed from the point combining middle and inner third of clavicle by progressing the needle under the clavicle with the needle tip pointing the upper notch of the sternum. The duration of the intervention was defined as the time between the beginning of the puncture and cannulation of the subclavian vein. The repeated intervention was defined as the removal of the needle from the puncture area completely and beginning the puncture from a different region. Arterial puncture was defined as the puncture of the subclavian artery accidentally. An independent observer calculated the duration of each successful venous puncture. Number of attempts for venous cannulation and number of arterial puncture was also recorded. In the roadmap group, after stabilization of the arm, a roadmap was formed by injecting $15 \mathrm{ml}$ of contrast agent through antecubital venous line [Figure 1]. The puncture was performed in the

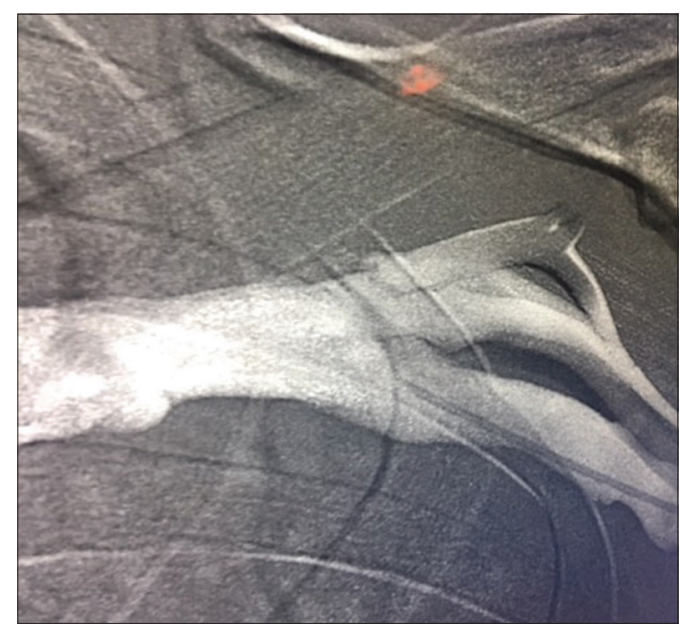

Figure 1: Roadmap-guided subclavian vein puncture 
same way as described above and time to puncture, number of attempts for venous cannulation and number of arterial puncture was also recorded. Fluoroscopy duration required for creating roadmap was recorded by an angiography technician. The total fluoroscopy time was also noted if fluoroscopy was needed in the conventional procedure group. After the implantation and fixation of the leads, the pocked irrigated with antibiotic (rifocine) and pacemaker generator was inserted into the pocket enabling that the portion of leads outside the vein are placed under the generator which was fixed by a single suture. Then, the incision was sutured and the pacemaker was programmed. Pressure was applied on to the incision area by 1000 cc physiological saline solution pack for $2 \mathrm{~h}$. Patients were followed up for 24-48 h. Any complication such as pneumothorax and pocket hematoma was recorded. Ten days later, incision area was reevaluated during the removal of the sutures, and any complication such as wound infection was recorded.

This study was approved by the Ethics Committee of the Kahramanmaras Sutcu Imam University under protocol number 52. All the procedures in this study were in accordance with the 1975 Helsinki Declaration, updated in 2013. Informed consent was obtained from all participants included in the study.

\section{Statistical analysis}

Statistical analyses were conducted through SPSS 17.0 (for Windows SPSS 17.0, Chicago, Illinois, USA). Continuous variables were presented as mean \pm standard deviation (for parameters with normal distribution) and median $(25 \% / 75 \%$ interquartile range) for parameters without normal distribution, and categorical variables were presented as percentages. Normality analysis was performed using the Kolmogorov-Smirnov test. Comparison of categorical variables between the groups was performed using the Chi-square test. The Student's $t$-test was used for the comparison of normally distributed variables, and the Mann-
Whitney U-test was used for the comparison of normally distributed multiple variables. A two-tailed $P<0.05$ was determined to be statistically significant within a confidence interval of $95 \%$.

\section{RESULTS}

\section{Randomization phase}

In study, 126 patients were enrolled consecutively and randomized (1:1) either to conventional group or roadmap group. One of the patients who received a single-chamber pacemaker with the roadmap method was excluded from the study because of withdrawing consent. Two patients in the conventional group were switched to roadmap group due to failure of subclavian vein puncture with conventional method. Accordingly, roadmap group composed of 64 patients and conventional group consisted of 61 patients. For a total of 125 devices, 245 leads were implanted. Operators received a deadline of $5 \mathrm{~min}$ or 8 subclavian vein puncture to complete the conventional method subclavian vein puncture.

Baseline characteristics of study population are presented in Table 1. Of these patients, 45 (36\%) underwent single chamber (SC) ICD/pacemaker, 40 (32\%) underwent dual chamber (DC) ICD/pacemaker, and 40 (32\%) underwent CRT implantations. Roadmap-guided implantation was performed in 64 patients (22 [35\%] SC ICD/pacemaker, 22 [34\%] DC ICD/pacemaker, and 20 [31\%] CRT) while the implantation was performed by conventional method in 61 patients $(23$ [37\%] SC ICD/pacemaker, 18 [30\%] DC ICD/pacemaker, and 20 [33\%] CRT). There was no significant difference between groups considering SC ICD/pacemaker, DC ICD/pacemaker, and CRT implantation ratios $(P=0.881, P=0.645$, and $P=0.759$, respectively). Demographic data of the patients were given in Table 1. Groups were similar for basal characteristic features. There was no statistically significant difference between groups considering hematological and biochemical parameters [Table 1].

\begin{tabular}{|c|c|c|c|c|}
\hline Baseline characteristics & All patients $(n=125)$ & Roadmap group ( $n=64)$ & Conventional group $(n=61)$ & $P$ \\
\hline Age, years* & $67(58 / 75)$ & $65(57 / 72)$ & $68(58.5 / 76)$ & 0.149 \\
\hline Weight, kg & $73.4 \pm 12.3$ & $72 \pm 11.1$ & $74.8 \pm 13.7$ & 0.198 \\
\hline Height, $\mathrm{cm}^{*}$ & $161(155 / 168)$ & $158(154.25 / 163.75)$ & $164.5(159.25 / 170.75)$ & $<0.001$ \\
\hline Gender, male/female, $n(\%)$ & $90 / 35(72 / 28)$ & $45 / 20(69.2 / 30.8)$ & $45 / 15(75 / 25)$ & 0.473 \\
\hline Hypertension, $n(\%)$ & $81(64.8)$ & $41(63.1)$ & $40(66.7)$ & 0.761 \\
\hline Diabetes mellitus, $n(\%)$ & $56(44.8)$ & $29(44.6)$ & $27(45)$ & 0.972 \\
\hline Current smoking, $n(\%)$ & $79(63.2)$ & $42(64.6)$ & $37(61.7)$ & 0.647 \\
\hline Hyperlipidemia, $n(\%)$ & $82(65.6)$ & $46(70.8)$ & $36(60)$ & 0.163 \\
\hline COPD, $n(\%)$ & $30(24)$ & $15(23.1)$ & $15(25)$ & 0.839 \\
\hline $\mathrm{CAD}, n(\%)$ & $95(76)$ & $49(75.4)$ & $46(76.7)$ & 0.989 \\
\hline $\mathrm{SC}$ pace or ICD, $n(\%)$ & $45(36)$ & $23(35.4)$ & $22(36.7)$ & 0.881 \\
\hline DC pace or ICD, $n(\%)$ & $40(32)$ & $22(33.8)$ & $18(30)$ & 0.645 \\
\hline CRT, $n(\%)$ & $40(32)$ & $20(30.8)$ & $20(33.3)$ & 0.759 \\
\hline
\end{tabular}

*Data presented as median (25/75\% IQR). CAD: Coronary artery disease, COPD: Chronic obstructive pulmonary disease, CRT: Cardiac resynchronization therapy, DC: Dual chamber, ICD: Implantable cardioverter defibrillator, SC: Single chamber, IQR: Interquartile range 
On the comparison of outcomes, it was seen that 186 punctures were performed in roadmap group and 332 punctures were done in conventional group. The median duration of intervention for each puncture was $27 \mathrm{~s}(15 / 46)$ in roadmap group and $56 \mathrm{~s}$ (30/100) in conventional group. The number of attempts for a successful puncture was detected as $1(1 / 2)$ in roadmap group and $2(2 / 4)$ in conventional group. Arterial puncture incidence was $10.3 \%$ in roadmap group and $37 \%$ in conventional group. Time to puncture, number of attempts for successful puncture and arterial puncture ratio was seen significantly lower in roadmap group $(P<0.001, P<0.001$, and $P<0.001$, respectively). When peri- or post-procedural complications were compared, incidence of pneumothorax and intramuscular puncture was seen lower significantly $(P=0.046$ and $P=0.006$, respectively). Although pocket hematoma was seen less frequently in roadmap group, the difference between groups was not statistically significant $(P=0.075)$. The groups were found similar as the success of the procedure $(P=0.113)$ [Table 2].

When mean duration of puncture, number of attempts for successful puncture and ratio of arterial puncture were compared according to device type, it was found that usage of roadmap method for device implantation reduced the time needed for successful puncture, number of attempts for successful puncture and incidence of arterial puncture significantly in all types of devices. In patients undergoing VR and DR pacemaker/ICD implantation, total procedure time was less in road map group whereas in patients performed CRT implantation, total procedure times were same in both groups. Considering fluoroscopy durations, images were taken from all patients by the way of fluoroscopy for $5 \mathrm{~s}$ in road map group. Then, puncture was performed and no additional fluoroscopy was performed. In conventional group, fluoroscopy was not performed in successful blinded punctures, but fluoroscopy times required for venography and anatomic localization were recorded. These durations were found as $20 \mathrm{~s}(0 / 40)$ for VR and $20 \mathrm{~s}(0 / 43)$ for DR and were significantly higher as compared to those of road map group ( $P=0.001$ for both). In CRT implantations, fluoroscopy times used for cannulation of coronary sinus were longer and were found similar between two groups ( $28.3 \pm 6.0$ vs. $30.7 \pm 8, P=0.286)$ [Table 3].

Table 2: Procedural characteristics and complication frequencies of both groups

\begin{tabular}{|c|c|c|c|}
\hline & Roadmap group ( $n=64)$ & Conventional group $(n=61)$ & $P$ \\
\hline Total/successful punctures, $n$ & $186 / 126$ & $332 / 119$ & $<0.001$ \\
\hline Failed attempts, $n(\%)$ & $60(32.3)$ & $213(64.2)$ & $<0.001$ \\
\hline Time to puncture, $\mathrm{s}^{*}$ & $27(15 / 46)$ & $56(30 / 100)$ & $<0.001$ \\
\hline Attempts for successful puncture, $n^{*}$ & $1(1 / 2)$ & $2(2 / 4)$ & $<0.001$ \\
\hline Incidence of arterial puncture, $\%$ & 10.3 & 37 & $<0.001$ \\
\hline Incidence of pneumothorax, $\%$ & 0.8 & 5 & 0.046 \\
\hline Incidence of intramuscular punctures, $\%$ & 0 & 5.9 & 0.006 \\
\hline Incidence of pocket hematoma, $\%$ & 1.6 & 5.9 & 0.075 \\
\hline
\end{tabular}

*Data presented as median (25/75\% IQR). IQR: Interquartile range

\section{Table 3: Procedural characteristics of both groups according to device types}

\begin{tabular}{|c|c|c|c|}
\hline & Roadmap group $(n=64)$ & Conventional group $(n=61)$ & $P$ \\
\hline SC ICD/pace, $n$ & 22 & 23 & - \\
\hline Attempts for successful puncture, $n^{*}$ & $1(1 / 2)$ & $3(1 / 4)$ & 0.004 \\
\hline Time to puncture, $\mathrm{s}^{*}$ & $30(23 / 70)$ & $145(18 / 180)$ & 0.014 \\
\hline Incidence of arterial puncture, $\%$ & 9.1 & 30.4 & 0.063 \\
\hline Fluoroscopy times, $\mathrm{s}^{*}$ & $5(5 / 5)$ & $20(0 / 40)$ & 0.001 \\
\hline Total procedure time, $\min ^{*}$ & $26 \pm 5.1$ & $30.6 \pm 7.2$ & 0.017 \\
\hline DC ICD/pace, $n$ & 22 & 18 & - \\
\hline Attempts for successful puncture, $n^{*}$ & $1(1 / 2)$ & $2(1.25 / 4)$ & $<0.001$ \\
\hline Time to puncture, $\mathrm{s}^{*}$ & $25(10 / 37.25)$ & $63.5(22.25 / 86.25)$ & $<0.001$ \\
\hline Incidence of arterial puncture, $\%$ & 9.1 & 33.3 & 0.006 \\
\hline Fluoroscopy times, $\mathrm{s}^{*}$ & $5(5 / 5)$ & $20(0-43)$ & 0.001 \\
\hline Total procedure time, min* & $33.7 \pm 5.2$ & $39.9 \pm 5.9$ & 0.001 \\
\hline CRT, $n$ & 20 & 20 & - \\
\hline Attempts for successful puncture, $n^{*}$ & $1(1 / 2)$ & $2(2 / 4)$ & $<0.001$ \\
\hline Time to puncture, $\mathrm{s}^{*}$ & $26(15 / 46)$ & $47(30 / 97)$ & $<0.001$ \\
\hline Incidence of arterial puncture, $\%$ & 11.7 & 40.3 & $<0.001$ \\
\hline Fluoroscopy times, min* & $28.3 \pm 6.0$ & $30.7 \pm 8$ & 0.286 \\
\hline Total procedure time, $\mathrm{min} *$ & $65(40 / 100)$ & $65(45 / 120)$ & 0.242 \\
\hline
\end{tabular}

*Data presented as median (25/75\% IQR). IQR: Interquartile range, DC: Dual chamber, SC: Single chamber, CRT: Cardiac resynchronization therapy, ICD: Implantable cardioverter defibrillator 


\section{Discussion}

This is the first study evaluating the effect of roadmap use on the success and the complication rate of the implantation of permanent pacemakers. The study revealed that complication rate was lower; success rate of puncture was higher; fluoroscopy time, total procedure time, and time to puncture were lower in patients undergoing permanent pacemaker implantation using roadmap technique. Since roadmap technique allows the anatomic imagination of subclavian vein, cannulation of extrathoracic portion of subclavian vein may prevent punctures through the muscle and decrease the probability of lead fractures.

Nowadays, the subclavian vein is preferred for permanent pacemaker implantation because of rapid and easy applicability. The number of attempts and time for successful puncture of subclavian vein depends on both anatomical factors and operator experience. A technique that can minimize the unfavorable effect of these two factors is supposed to decrease both the rate of puncture failure and duration of successful puncture. Accordingly, this will reduce the complications related with puncture and increase patient comfort because of less number of trials for successful puncture and shorter procedure duration. Previously, Higano et al. and Chan et al. performed subclavian puncture by the guidance of fluoroscopic venography in their studies and reported that puncture guided by venography was safer. ${ }^{[6,7]}$ Similar to these studies, we planned to compare subclavian puncture performed after anatomic imagination of subclavian vein with conventional blinded puncture. But differently, we used roadmap technique to visualize subclavian vein. There are several advantages of roadmap technique to venography. One of them is shortened fluoroscopy duration and accordingly decreased exposure of both patient and operator to radiation. The other one is that single roadmap image enables opportunity to multiple attempts of puncture; so recurrent use of contrast agent during the implantation of pacemakers with multiple leads is avoided. However, in the group of patients performed conventional method, there is no need for fluoroscopy and contrast injection during successful venous puncture. In case of contrast agent allergy or renal impairment, blinded puncture is superior to roadmap or fluoroscopy-guided techniques. On the other hand, when the intervention is considered under fluoroscopic venography due to anatomical difficulties or unsuccessful attempts, then the use of roadmap method may be more reasonable unless there is no contraindication to contrast agents.

Although various studies comparing different techniques used for intervention are present in the literature, our study is the first one using roadmap technique for subclavian puncture. ${ }^{[8,9,12,13]} \mathrm{In}$ this study, blinded subclavian venous puncture was compared with the puncture guided by roadmap with regard to success of the procedure, time needed for a successful venous puncture and unintended puncture of subclavian artery, and roadmap technique was found superior to blinded puncture in all these parameters. Main complications of subclavian venous puncture are pneumothorax and hemothorax, and their incidence is $1 \%-3 \%{ }^{[14]}$ In order to decrease these complications venography has been used frequently and shown to be effective in various studies. ${ }^{[6,15,16]}$ In our study, pneumothorax was reported only in the blinded puncture group. Higher number of attempts for successful puncture in this group may be a causative factor for these complications.

Pocket hematoma is a widely seen complication of pacemaker implantation. ${ }^{[17]}$ It increases the duration of hospitalization and the risk of device infection. Many factors may contribute to development of pocket hematoma. Procedure type (first implantation or reimplantation), operator experience, device size, number of leads, venous route (subclavian or cephalic vein), body mass index and anticoagulant and/ or antiaggregant use are regarded among them. ${ }^{[18-22]}$ In our study, pocket hematoma was shown to be more frequent in the group of blinded puncture. Detailed analysis revealed that unintended puncture of subclavian artery was performed in most of the patients having pocket hematoma. This finding made us to think that subclavian arterial puncture could be a contributing factor for pocket hematoma. However, further studies are needed to support this hypothesis considering the factors predisposing to pocket hematoma such as device size, number of leads implanted, and patient's medication.

Another problem that can be encountered during subclavian puncture is puncture of intrathoracic subclavian vein that can result in lead fracture. Intrathoracic subclavian puncture causes trapping of leads between costoclavicular ligament and/or subclavian muscles which results in lead fractures. ${ }^{[5]}$ Based on these data, we intended to puncture extrathoracic subclavian vein in the patients of roadmap group. In blinded puncture, since we could not do this discrimination, there were some intramuscular punctures, which were identified by the factors based on the operator experience such as resistance to the insertion of sheath and resistance and difficulty during manipulation of leads after removal of sheath. Puncture site was changed in these patients to decrease the risk of lead fracture. Roadmap-guided puncture may provide to avoid intramuscular puncture and/or intrathoracic puncture of subclavian vein, which is the most important cause of lead fractures, independently of operator experience. As a result, lead fractures can be prevented by this technique.

\section{Study limitations}

Our study has some limitations. Of these, number of study population was relatively small and all pacemakers were implanted by electrophysiologists. The roadmap technique has also some limitations such as keeping the patient immobile and use of contrast agents for visualization of the vein. Although we thought that lead fractures might be prevented by puncture of extrathoracic subclavian vein, we could not document the rate of lead fracture since long-term follow-up was not done. Finally, the team conducting the study will also report the results. This is an important limitation for bias. 


\section{Conclusion}

Our study revealed that number of attempts for venous puncture was lower and time needed for successful puncture was shorter in patients undergoing pacemaker implantation by roadmap technique. Moreover, complications of pacemaker implantation such as pneumothorax, hemothorax, and pocket hematoma were seen less frequently in roadmap group. These data indicate that procedure of pacemaker implantation can be managed more successfully and safely using roadmap technique.

\section{Financial support and sponsorship}

Nil.

\section{Conflicts of interest}

There are no conflicts of interest.

\section{References}

1. Carrión-Camacho MR, Marín-León I, Molina-Doñoro JM, González-López JR. Safety of permanent pacemaker implantation: A prospective study. J Clin Med 2019;8. pii: E35.

2. van Rugge FP, Savalle LH, Schalij MJ. Subcutaneous single-incision implantation of cardioverter-defibrillators under local anesthesia by electrophysiologists in the electrophysiology laboratory. Am J Cardiol 1998;81:302-5.

3. Israel CW, Ekosso-Ejangue L. Problems, complications, and emergencies during pacemaker implantation. Importance of access. Herzschrittmacherther Elektrophysiol 2015;26:309-19.

4. Sassard T, Fareh S, Farhat F. An unusual complication of a ventricular pace maker electrode. Eur J Cardiothorac Surg 2007;31:306.

5. Magney JE, Flynn DM, Parsons JA, Staplin DH, Chin-Purcell MV, Milstein S, et al. Anatomical mechanisms explaining damage to pacemaker leads, defibrillator leads, and failure of central venous catheters adjacent to the sternoclavicular joint. Pacing Clin Electrophysiol 1993;16:445-57.

6. Higano ST, Hayes DL, Spittell PC. Facilitation of the subclavian-introducer technique with contrast venography. Pacing Clin Electrophysiol 1990;13:681-4.

7. Chan NY, Liem LB, Mok NS, Wong W. Corrigendum to "Clinical experience of contrast venography guided axillary vein puncture in biventricular pacing" [Int. J. Cardiol 92 (2003) 55-58]. Int J Cardiol 2017;227:957.

8. Harada Y, Katsume A, Kimata M, Hikosaka T, Yamanaka S, Akashi K, et al. Placement of pacemaker leads via the extrathoracic subclavian vein guided by fluoroscopy and venography in the oblique projection. Heart Vessels 2005;20:19-22.
9. Nakata A, Harada T, Kontani K, Hirota S. Extrathoracic subclavian venipuncture by using only the J-type guidewire for permanent pacemaker electrode placement. Int Heart J 2013;54:129-32.

10. Gyánó M, Góg I, Óriás VI, Ruzsa Z, Nemes B, Csobay-Novák C, et al. Kinetic imaging in lower extremity arteriography: Comparison to digital subtraction angiography. Radiology 2019;290:246-53.

11. Calkins H, Ramza BM, Brinker J, Atiga W, Donahue K, Nsah E, et al. Prospective randomized comparison of the safety and effectiveness of placement of endocardial pacemaker and defibrillator leads using the extrathoracic subclavian vein guided by contrast venography versus the cephalic approach. Pacing Clin Electrophysiol 2001;24:456-64.

12. Mehrotra S, Rohit MK. Prospective study to develop surface landmarks for blind axillary vein puncture for permanent pacemaker and defibrillator lead implantation and compare it to available contrast venography guided technique. Indian Heart J 2015;67:136-40.

13. Clark BC, Janson CM, Nappo L, Pass RH. Ultrasound-guided axillary venous access for pediatric and adult congenital lead implantation. Pacing Clin Electrophysiol 2019;42:166-70.

14. Pinelli F, Barbani F, Pittiruti M. Ultrasound-guided cannulation of the subclavian vein - An update. N Engl J Med 2018;379:e27.

15. Lalu MM, Fayad A, Ahmed O, Bryson GL, Fergusson DA, Barron CC, et al. Ultrasound-guided subclavian vein catheterization: A systematic review and meta-analysis. Crit Care Med 2015;43:1498-507.

16. Martin-Casañas FV, Caballero-Estevez N, Dominguez-Rodriguez A, Abreu-Gonzalez P, Laynez-Cerdeña I. Cardiac device infections is associated with pocket hematoma and diabetes mellitus: The role of the cardiovascular nurse. Int J Cardiol 2014;171:e5-7.

17. Nowak B, Tasche K, Barnewold L, Heller G, Schmidt B, Bordignon $\mathrm{S}$, et al. Association between hospital procedure volume and early complications after pacemaker implantation: Results from a large, unselected, contemporary cohort of the german nationwide obligatory external quality assurance programme. Europace 2015;17:787-93.

18. Korantzopoulos P, Letsas KP, Liu T, Fragakis N, Efremidis M, Goudevenos JA, et al. Anticoagulation and antiplatelet therapy in implantation of electrophysiological devices. Europace 2011;13:1669-80.

19. Birnie DH, Healey JS, Wells GA, Verma A, Tang AS, Krahn AD, et al. Pacemaker or defibrillator surgery without interruption of anticoagulation. N Engl J Med 2013;368:2084-93.

20. Dai Y, Chen KP, Hua W, Zhang JT, Zhang S. Dual antiplatelet therapy increases pocket hematoma complications in chinese patients with pacemaker implantation. J Geriatr Cardiol 2015;12:383-7.

21. Guo JP, Shan ZL, Guo HY, Yuan HT, Lin K, Zhao YX, et al. Impact of body mass index on the development of pocket hematoma: A retrospective study in Chinese people. J Geriatr Cardiol 2014;11:212-7.

22. Migliore F, Curnis A, Bertaglia E. Axillary vein technique for pacemaker and implantable defibrillator leads implantation: A safe and alternative approach? J Cardiovasc Med (Hagerstown) 2016;17:309-13. 